\title{
Web-interfaced Nonlinear Optical Waveguide and Photonic Crystal Simulator

\author{
DE-FG02-01ER83145
}

\author{
Phase I Final Report \\ S. Enguehard \\ B. Hatfield \\ Applied Mathematical Physics Research, Inc. \\ Lexington, MA
}

\section{Introduction and Overview}

An integrated optics design tool will reduce the design cost of photonic components while speeding up the design process. It will expand the availability of this technology to more specialized applications such as custom purpose sensors. At the heart of any integrated optics simulator and design tool is an accurate beam propagation model and simulation. The goal of the Phase I project was to investigate new techniques and viewpoints for computing the propagation of light through photonic crystals and waveguides. We report here on the development of new methods for the computation of spectral bandpass properties of photonic crystals and for the electromagnetic wave propagation in second order nonlinear optical waveguides. The former is based on a generalization of characteristic matrices while the latter is based on path integrals.

\section{Photonic Crystals}

There exist some "established" approaches to numerically solve the crystal propagation equations. One popular method to determine the band structure involves solving the wave equation for the magnetic field derived from Maxwell's field equations[3]. The wave equation is written as an eigenvalue equation. Due to the periodicity of the dielectric, one naturally wants to Fourier transform everything in sight. The Fourier transform guarantees that the field is properly periodic without explicitly applying boundary conditions. The differential operator becomes a matrix whose eigenvalues and eigenmodes are found numerically. Fortunately the differential operator is 
Hermitian so that the numerics are easier and the modes orthonormal. Unfortunately, the dielectric profile usually contains sharp discontinuities. This means that the high frequency components of the Fourier transform are important, and numerical convergence is slow and sometimes tricky (marginally stable or unstable). Furthermore, the matrix depends on the wave vector $\vec{k}$ and the numerical diagonalization must be repeated for each value of $\vec{k}$ in the first Brillouin zone (which may be further reduced by discrete symmetries present in the dielectric pattern).

The wave equation for the electric field derived from Maxwell's equation is the Helmholtz equation, and that for the magnetic field mentioned above is the magnetic equivalent. Inside any region of constant dielectric, we know exactly the solutions to these equations. They are plane waves but they do not have wave vectors that are associated with the reciprocal lattice, that is, they do not appear in the Fourier expansion. Thus by Fourier transforming everything, we are covering up the fact that we know the solution. The Fourier coefficients that are found by diagonalizing the matrix are those of a plane wave. The magnitude of the wave vector for the plane wave solution is set by the value of the dielectric through the Helmholtz equation. The direction of the wave vector, as well as the amplitude of the plane wave, is set by phase matching at the dielectric boundaries, and by applying the Maxwell boundary conditions at dielectric interfaces (e.g. the tangential component of the electric field is continuous across the surface, etc.). The phase matching turns the plane waves into Bloch waves. The eigenvalues and eigenmodes are determined by requiring periodicity. In summary, the magnitude of the wave vector is set by the value of the dielectric, but its direction is determined by the boundary conditions at the interfaces, and the periodicity.

To avoid just duplicating previous work, and in order to gain physical insight and expand the analytical basis, we purposely worked outside of this envelope. We have been successful at developing new calculational frameworks for photonic crystals based on characteristic matrices, and therefore were able to obtain some analytical results, and a new framework for computation. In this framework, we avoid Fourier transformations, and in particular we avoid Fourier transforming the dielectric profile. Thus sharp discontinuities in the dielectric profile present no numerical problems.

To summarize our new results, we impose periodicity on the characteristic matrix for the unit cell by requiring that the characteristic matrix or propa- 
gator reproduce the input electric and magnetic fields up to a phase. In this way, Bloch waves are naturally incorporated into the framework. Using the fact that the characteristic matrix or propagator is unimodular (no absorption), we than find that the spectral equation for the crystal band structure is given by the trace of the characteristic matrix.

For example, let bf $\mathrm{G}$ be the characteristic matrix that propagates in the $z$-direction the electromagnetic field polarized in the $y$-direction:

$$
\left(\begin{array}{l}
E_{y}(z) \\
H_{x}(z)
\end{array}\right)=\mathbf{G}(z)\left(\begin{array}{c}
E_{y}(0) \\
H_{x}(0)
\end{array}\right) \text {. }
$$

If the period in the $z$-direction is $\Lambda$ and $k$ is the wave vector, then periodicity requires that

$$
\left(\begin{array}{c}
E_{y}(\Lambda) \\
H_{x}(\Lambda)
\end{array}\right)=\mathbf{G}(\Lambda)\left(\begin{array}{c}
E_{y}(0) \\
H_{x}(0)
\end{array}\right)=\exp (i k \Lambda)\left(\begin{array}{c}
E_{y}(0) \\
H_{x}(0)
\end{array}\right) .
$$

There will be a nontrivial solution only if

$$
\operatorname{det}|\mathbf{G}-\exp (i k \Lambda) \mathbf{1}|=0 \text {. }
$$

Since $\mathbf{G}$ is unimodular, eq.(3) yields the spectral equation:

$$
\frac{1}{2} \operatorname{Tr} \mathbf{G}=\cos (k \Lambda)
$$

\section{1-D Crystals}

For simplicity, and for illustration, we begin with 1-D photonic crystals. One can also find the 1-D problem embedded in the analysis of the 2-D photonic crystal. We will derive the spectral equation from the characteristic matrix.

Let the 1-D photonic crystal consist of alternating layers of thin slabs with differing dielectric constants $\epsilon_{1}$ and $\epsilon_{2}$. As illustrated in Figure 1, the slabs are parallel to the $x y$-plane, the $z$-axis is normal to the slabs, the width of the first slab is $a$, and the period in the $z$-direction is $\Lambda$. The analysis can easily be generalized to handle unit "cells" consisting of more than 2 layers. The three layer result is given at the end of this section.

We will first solve for spectral equation by solving the Helmholtz equation and applying the boundary conditions. This will demonstrate that the 


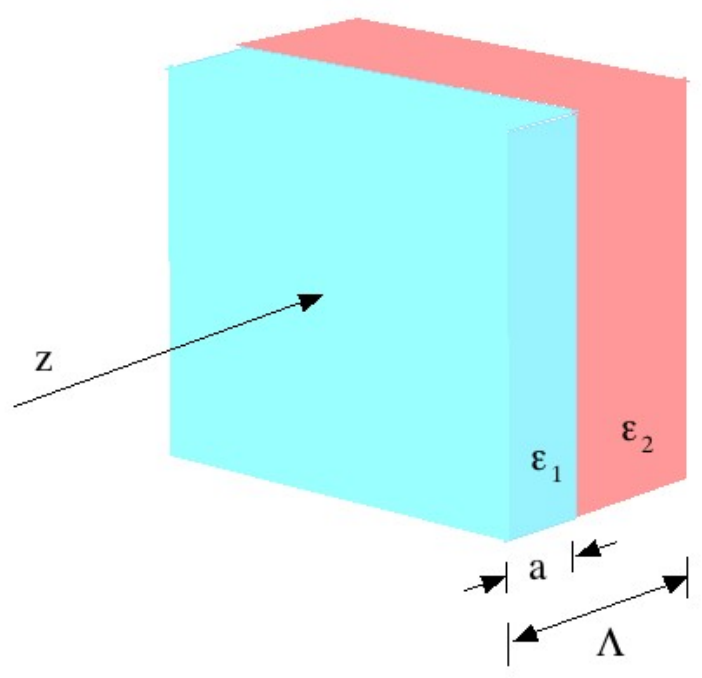

Figure 1: Unit cell of a simple 1-D photonic crystal.

charateristic matrix method is simpler and more elegant. Without loss of generality, let the electric field be polarized in the $y$-direction.. For $0<z<a$, the electric field satisfies

$$
\left(\partial_{z}^{2}+\kappa_{1}^{2}\right) E_{y}(z)=0
$$

where $\kappa_{1}^{2}=\kappa^{2} \epsilon_{1}$ with $\kappa=\omega / c$. . The magnetic field is obtained from the electric field using

$$
\partial_{z} E_{y}=-\frac{i}{\kappa} \kappa_{1}^{2} H_{x}
$$

For the region $a-\Lambda<z<0$, substitute $\kappa_{2}^{2}=\kappa^{2} \epsilon_{2}$ for $\kappa_{1}^{2}$. The solution for $0<z<a$ is

$$
E_{y}(z)=A \exp \left(i \kappa_{1} z\right)+B \exp \left(-i \kappa_{1} z\right)
$$

and for $a-\Lambda<z<0$,

$$
E_{y}(z)=C \exp \left(i \kappa_{2} z\right)+D \exp \left(-i \kappa_{2} z\right)
$$

Written in Bloch wave form, we have

$$
E_{y}(z)=\exp (i k z) U(z)
$$


where $U(z)$ is periodic with crystal period $\Lambda, U(\Lambda+z)=U(z)$. In order to determine the spectral equation, we apply the Maxwell boundary conditions (continuous tangential electric and magnetic fields) at $z=0$ and $z=a$. At $z=0$ we find

$$
\begin{aligned}
A+B & =C+D \\
i\left(\kappa_{1}-k\right) A-i\left(\kappa_{1}+k\right) B & =i\left(\kappa_{2}-k\right) C-i\left(\kappa_{2}+k\right) D
\end{aligned}
$$

and at $z=a$ we have

$$
\begin{gathered}
\exp \left(i\left(\kappa_{1}-k\right) a\right) A+\exp \left(-i\left(\kappa_{1}+k\right) a\right) B= \\
\exp \left(i\left(\kappa_{2}-k\right)(\Lambda-a)\right) C+\exp \left(-i\left(\kappa_{2}+k\right)(\Lambda-a)\right) D \\
i\left(\kappa_{1}-k\right) \exp \left(i\left(\kappa_{1}-k\right) a\right) A-i\left(\kappa_{1}+k\right) \exp \left(-i\left(\kappa_{1}+k\right) a\right) B= \\
i\left(\kappa_{2}-k\right) \exp \left(i\left(\kappa_{2}-k\right)(\Lambda-a)\right) C-i\left(\kappa_{2}+k\right) \exp \left(-i\left(\kappa_{2}+k\right)(\Lambda-a)\right) D .
\end{gathered}
$$

Eqs.(10) and (11) provide four equations with four unknowns and no source terms. A nontrivial solution exists only if the determinant of the coefficients vanishes. This condition generates the spectral equation. After a considerable amount of algebra, we have

$$
\cos \left(\kappa_{1} a\right) \cos \left(\kappa_{2}(\Lambda-a)\right)-\left(\frac{\kappa_{1}^{2}+\kappa_{2}^{2}}{2 \kappa_{1} \kappa_{2}}\right) \sin \left(\kappa_{1} a\right) \sin \left(\kappa_{2}(\Lambda-a)\right)=\cos (k \Lambda) .
$$

Recall that $\omega / c=\kappa$ and that $\kappa^{2} \epsilon_{1}=\kappa_{1}^{2}$ and $\kappa^{2} \epsilon_{2}=\kappa_{2}^{2}$. Thus the left-hand side of eq.(12) can be thought of as a function of $\omega$, while the right-hand side depends the selected (initial) value for the wave vector $k$. Clearly the right-hand side of eq.(12) can not be bigger than 1 or less than -1 . The values of $\omega$ that cause the left-hand side to be bigger than 1 or less than -1 reside in the photonic band gaps.

Now imagine a unit cell made of three or more layers. Each layer would add two more unknowns. While the matrix of coefficients would contain some vanishing elements, the computation of the determinant rapidly becomes more involved. We will next generate the spectral equation using characteristic matrices. The inclusion of more layers merely involves matrix multiplication. As we will see, the spectral equation for mult-layers is straightforward to generate. 
Consider again propagation in the direction of the $z$-axis. For the electric field along the $y$-direction, and the magnetic field along the $x$-direction, Maxwell's equations yield

$$
\begin{aligned}
\partial_{z}\left(\begin{array}{c}
E_{y} \\
H_{x}
\end{array}\right) & =\left(\begin{array}{cc}
0 & -i \omega / c \\
-i \omega \epsilon(z) / c-i c / \omega \partial_{x}^{2} & 0
\end{array}\right)\left(\begin{array}{c}
E_{y} \\
H_{x}
\end{array}\right) \\
& =-\frac{i}{\kappa}\left(\begin{array}{cc}
0 & \kappa^{2} \\
\kappa^{2} \epsilon(z) & 0
\end{array}\right)\left(\begin{array}{c}
E_{y} \\
H_{x}
\end{array}\right),
\end{aligned}
$$

where, as before, we have $\omega / c=\kappa$, and, in the first layer, $\kappa^{2} \epsilon_{1}=\kappa_{1}^{2}$. Under these conditions, the solution to eq.(13) is

$$
\left(\begin{array}{l}
E_{y}(z) \\
H_{x}(z)
\end{array}\right)=\exp (\mathbf{H} z)\left(\begin{array}{c}
E_{y}(0) \\
H_{x}(0)
\end{array}\right) ; \quad \mathbf{H}=-\frac{i}{\kappa}\left(\begin{array}{cc}
0 & \kappa^{2} \\
\kappa_{1}^{2} & 0
\end{array}\right) .
$$

The exponential of the matrix $\mathbf{H}$, that is, the characteristic matrix $\mathbf{G}_{0}$, may be calculated explicitly using the series expansion for the exponential:

$$
\begin{aligned}
\mathbf{G}_{0}(z) & =\mathbf{1}+\mathbf{H} z+\frac{1}{2 !} \mathbf{H}^{2} z^{2}+\ldots \\
& =\left(\begin{array}{cc}
\cos \left(\kappa_{1} z\right) & -i \frac{\kappa}{\kappa_{1}} \sin \left(\kappa_{1} z\right) \\
-i \frac{\kappa_{1}}{\kappa} \sin \left(\kappa_{1} z\right) & \cos \left(\kappa_{1} z\right)
\end{array}\right) .
\end{aligned}
$$

As usual, if we stack the layers together, the characteristic matrix for the entire set of layers is the matrix product of the characteristic matrices for the individual layers:

$$
\mathbf{G}(d)=\mathbf{G}_{0}\left(d-z_{N}\right) \mathbf{G}_{0}\left(z_{N}-z_{N-1}\right) \cdots \mathbf{G}_{0}\left(z_{1}\right) .
$$

Thus, the characteristic matrix for the unit cell of our 1-D photonic crystal is

$$
\mathbf{G}(\Lambda)=\mathbf{G}_{0}(\Lambda-a) \mathbf{G}_{0}(a)=\left(\begin{array}{ll}
g_{11} & g_{12} \\
g_{21} & g_{22}
\end{array}\right) .
$$

where

$$
\begin{aligned}
& g_{11}=\cos \left(\kappa_{1} a\right) \cos \left(\kappa_{2}(\Lambda-a)\right)-\frac{\kappa_{1}}{\kappa_{2}} \sin \left(\kappa_{1} a\right) \sin \left(\kappa_{2}(\Lambda-a)\right) \\
& g_{12}=-i \frac{\kappa}{\kappa_{1}} \sin \left(\kappa_{1} a\right) \cos \left(\kappa_{2}(\Lambda-a)\right)-i \frac{\kappa}{\kappa_{2}} \sin \left(\kappa_{2}(\Lambda-a)\right) \cos \left(\kappa_{1} a\right) \\
& g_{21}=-i \frac{\kappa_{1}}{\kappa} \sin \left(\kappa_{1} a\right) \cos \left(\kappa_{2}(\Lambda-a)\right)-i \frac{\kappa_{2}}{\kappa} \sin \left(\kappa_{2}(\Lambda-a)\right) \cos \left(\kappa_{1} a\right) \\
& g_{22}=\cos \left(\kappa_{1} a\right) \cos \left(\kappa_{2}(\Lambda-a)\right)-\frac{\kappa_{2}}{\kappa_{1}} \sin \left(\kappa_{1} a\right) \sin \left(\kappa_{2}(\Lambda-a)\right)
\end{aligned}
$$


Eq.(17) is the characteristic matrix for a unit cell of 2 layers. The photonic crystal consists of a large stack of the 2-layer units, hence the total characteristic matrix is the matrix eq.(17) raised to a large power. None the less, we can derive the spectral equation and determine the band gaps from eq.(17) using the method outlined in eqs.(2)-(4). The periodicity in the $z$-direction requires that the electric and magentic fields also be periodic, up to a phase, with the same period $\Lambda$. If the wave vector in the $\mathrm{z}$ direction is $k$, then

$$
\left(\begin{array}{c}
E_{y}(z) \\
H_{x}(z)
\end{array}\right)=\mathbf{G}(\Lambda)\left(\begin{array}{c}
E_{y}(0) \\
H_{x}(0)
\end{array}\right)=\exp (i k \Lambda)\left(\begin{array}{c}
E_{y}(0) \\
H_{x}(0)
\end{array}\right) .
$$

There will only be a solution for eq.(19) for nontrivial electric and magnetic fields if

$$
\operatorname{det}\left|\begin{array}{cc}
g_{11}-\exp (i k \Lambda) & g_{12} \\
g_{21} & g_{22}-\exp (i k \Lambda)
\end{array}\right|=0 .
$$

A well-known property of the characteristic matrix is that it is unimodular, i.e. $\operatorname{det} \mathbf{G}(\Lambda)=1$ (required by the reciprocity symmetry of Maxwell's equations). Making use of this fact, eq.(20) reduces to the spectral equation:

$$
\frac{g_{11}+g_{22}}{2}=\cos (k \Lambda) \text {. }
$$

The trace of the characteristic matrix is twice the real part of the Bloch wave phase factor. Plugging in the explicit expression for the matrix elements in eq.(18) into eq.(21), we arrive a the familiar result:

$$
\cos \left(\kappa_{1} a\right) \cos \left(\kappa_{2}(\Lambda-a)\right)-\frac{1}{2}\left(\frac{\kappa_{1}}{\kappa_{2}}+\frac{\kappa_{2}}{\kappa_{1}}\right) \sin \left(\kappa_{1} a\right) \sin \left(\kappa_{2}(\Lambda-a)\right)=\cos (k \Lambda),
$$

which, of course, is identical to eq.(12). Once again, the left-hand side of eq.(22) can be thought of as a function of $\omega=\kappa c$, while the right-hand side depends the selected value for the wave vector $k$. The values of $\omega$ that cause the left-hand side to be bigger than 1 or less than -1 reside in the photonic band gaps.

Once the values of $\omega$ for each band that solve eq.(22) for a given $k$ are determined, the corresponding values of $\kappa, \kappa_{1}$ and $\kappa_{2}$ can be inserted into eq.(19), and the equation solved for the corresponding eigenmodes of the electric and magnetic fields. One will find that the eigenmodes are just 
plane waves, but not Fourier modes. Note that we did not use a Fourier transform in this analysis. Periodicity was enforced with boundary conditions in eq.(19). These reults can be carried over to the 2-D case.

To demonstrate the ease of use of the characteristic matrix method, we will it analytically compute the spectral equation for a 3-layer unit cell. Let the dielectric coefficients of the three layers be $\epsilon_{1}, \epsilon_{2}$, and $\epsilon_{3}$ with widths $a$, $b$, and $\Lambda-(a+b)$ respectively. The characteristic matrix is $\mathbf{G}\left(\kappa_{3}, \Lambda-(a+\right.$ b)) $\mathbf{G}\left(\kappa_{2}, b\right) \mathbf{G}\left(\kappa_{1}, a\right)$. the result of the matrix multiplication of the right 2 matrices is given by eq.(18) with $\Lambda-a$ replaced by $b$. This result is then multiplied by eq.(15) with $\kappa_{1} \rightarrow \kappa_{3}$ and $z$ replaced by $\Lambda-(a+b)$. We, in fact, only need the diagonal elements to compute the trace. The resulting spectral equation is

$$
\begin{gathered}
\cos \left(\kappa_{3}(\Lambda-a-b)\right)\left[\cos \left(\kappa_{1} a\right) \cos \left(\kappa_{2} b\right)-\frac{1}{2}\left(\frac{\kappa_{1}}{\kappa_{2}}+\frac{\kappa_{2}}{\kappa_{1}}\right) \sin \left(\kappa_{1} a\right) \sin \left(\kappa_{2} b\right)\right] \\
-\sin \left(\kappa_{3}(\Lambda-a-b)\right)\left[\frac{1}{2}\left(\frac{\kappa_{1}}{\kappa_{3}}+\frac{\kappa_{3}}{\kappa_{1}}\right) \sin \left(\kappa_{1} a\right) \cos \left(\kappa_{2} b\right)\right. \\
\left.+\frac{1}{2}\left(\frac{\kappa_{2}}{\kappa_{3}}+\frac{\kappa_{3}}{\kappa_{2}}\right) \cos \left(\kappa_{1} a\right) \sin \left(\kappa_{2} b\right)\right]=\cos (k \Lambda) .
\end{gathered}
$$

\section{2-D Crystals}

The method outlined in eqs.(1)-(4) for determining the spectral equations using characteristic matrices and applied to 1-D crystals in the previous section carries directly over to higher dimensional crystals. For illustration, and to demonstrate explicitly that this method reduces the computation for the 2-D crystal to solving two 1-D crystals, consider the 2-D crystal given in Figure 2 . The crystal is periodic in the $x$-direction with period $\Lambda^{\prime}$ and periodic in the $z$-direction with period $\Lambda$. The crystal consist of rectangular columns of dielectric with alternating dielectric coefficients of $\epsilon_{1}$ and $\epsilon_{2}$. Without loss of generality we will take $\epsilon_{1}>\epsilon_{2}$, and denote $\epsilon_{1}$ as $\epsilon_{H}$ (high), and $\epsilon_{2}$ as $\epsilon_{L}$ (low).

Consider the first layer of the unit cell between $z=0$ and $z=a$. By itself, this layer is identical to a volume grating, and the dependence of the characteristic matrix for propagation from $z=0$ to $z=a$ is the same in the grating as in the crystal. In previous work, we discovered the generalization of the characteristic matrix for uniform layers to volume gratings, and call these grating characteristic matrices. 


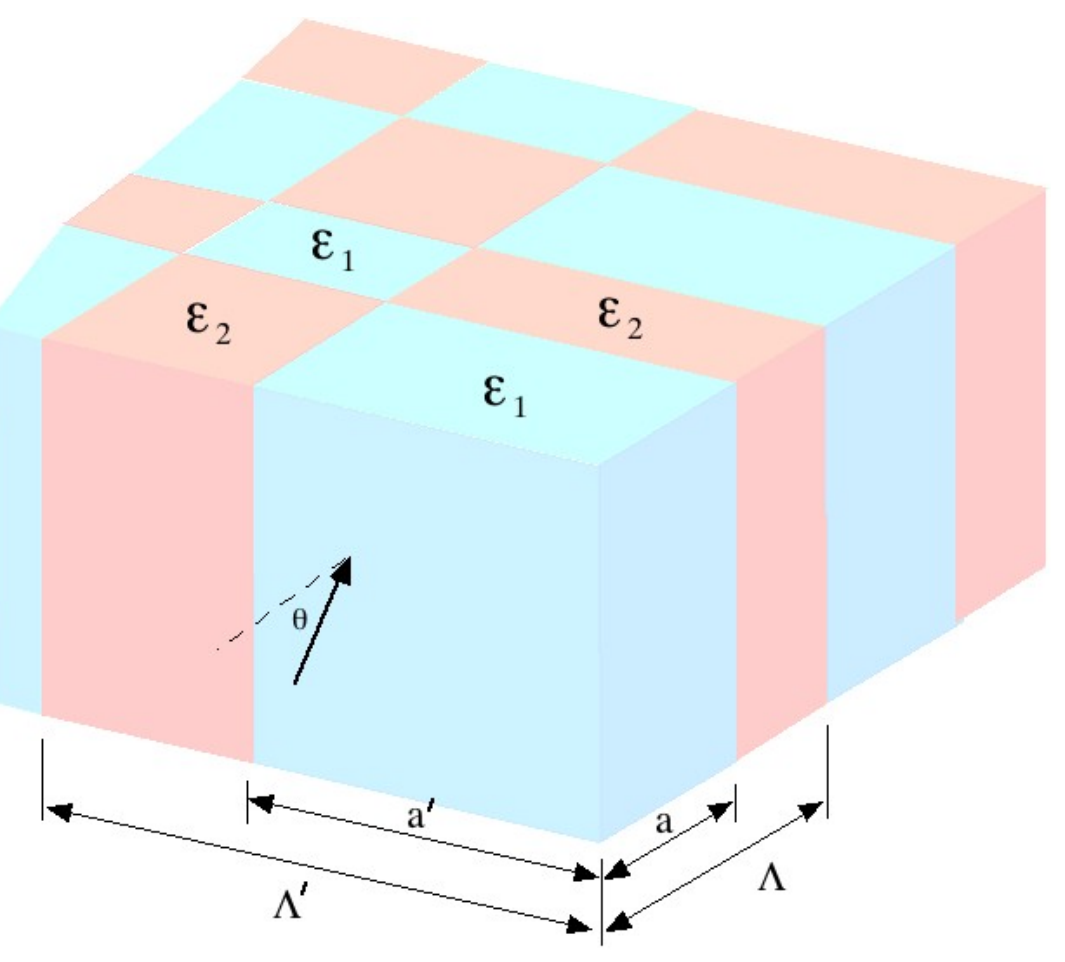

Figure 2: Unit cell of a simple 2-D photonic crystal.

Let us first work with TE polarization, $\vec{E}(x, z)=E_{y}(x, z) \widehat{y}$. The electric and magnetic fields satisfy Maxwell's equations, which we write in a form similar to eq.(13):

$$
\partial_{z}\left(\begin{array}{c}
E_{y} \\
H_{x}
\end{array}\right)=\mathbf{H}_{\mathrm{TE}}\left(\begin{array}{c}
E_{y} \\
H_{x}
\end{array}\right),
$$

where

$$
\mathbf{H}_{\mathrm{TE}}=\frac{i}{\kappa}\left(\begin{array}{cc}
0 & -\kappa^{2} \\
\mathbf{H}_{1} & 0
\end{array}\right),
$$

and

$$
\mathbf{H}_{1}=-\partial_{x}^{2}-\kappa^{2} \epsilon(x) .
$$

The solution to eq.(24) is

$$
\left(\begin{array}{l}
E_{y}(z) \\
H_{x}(z)
\end{array}\right)=\exp \left(\mathbf{H}_{\mathrm{TE}} z\right)\left(\begin{array}{c}
E_{y}(0) \\
H_{x}(0)
\end{array}\right) .
$$


Let $\left|\lambda_{n}\right\rangle$ be an eigenvector of $\mathbf{H}_{T E}$ with eigenvalue $\lambda_{n}$ and $\left|\alpha_{n}\right\rangle$ be an eigenvector of $\mathbf{H}_{1}$ with eigenvalue $\alpha_{n}$. That is,

$$
\mathbf{H}_{T E}\left|\lambda_{n}\right\rangle=\lambda_{n}\left|\lambda_{n}\right\rangle, \quad \mathbf{H}_{1}\left|\alpha_{n}\right\rangle=\alpha_{n}\left|\alpha_{n}\right\rangle .
$$

Plugging eq.(25) into eq.(28), we find that $\lambda_{n}= \pm \sqrt{\alpha_{n}}$ and

$$
\left|\lambda_{n}^{+}\right\rangle=\left(\begin{array}{c}
\left|\alpha_{n}\right\rangle \\
(i / \kappa) \sqrt{\alpha_{n}}\left|\alpha_{n}\right\rangle
\end{array}\right), \quad\left|\lambda_{n}^{-}\right\rangle=\left(\begin{array}{c}
\left|\alpha_{n}\right\rangle \\
-(i / \kappa) \sqrt{\alpha_{n}}\left|\alpha_{n}\right\rangle
\end{array}\right) .
$$

Thus, the eigenvector of $\mathbf{H}_{T E}$ can be written in terms of the eigenvectors of $\mathbf{H}_{1}$. The eigenvectors $\left|\lambda_{n}^{ \pm}\right\rangle$, i.e $\left|\alpha_{n}\right\rangle$, are the internal ("grating") modes for the layer between 0 and $z$. The combination $\exp \left(\lambda_{n} z\right)\left|\lambda_{n}\right\rangle$ is a solution of Maxwell's equations, as can be seen by direct substitution into eq.(24).

From eq.(27) we see that the characteristic matrix for the first layer of the crystal is

$$
\mathbf{G}_{\mathrm{TE}}(a)=\exp \left(\mathbf{H}_{\mathrm{TE}} a\right)
$$

Following a procedure similar to eq.(15), we find [1]

$$
\mathbf{G}_{\mathrm{TE}}=\left(\begin{array}{ll}
\mathbf{G}_{1} & \mathbf{G}_{2} \\
\mathbf{G}_{3} & \mathbf{G}_{1}
\end{array}\right)
$$

where each submatrix is expressed as a sum over the eigenmodes $\left|\alpha_{n}\right\rangle$ :

$$
\begin{aligned}
\mathbf{G}_{1}(a) & =\sum_{n} \cosh \left(\sqrt{\alpha_{n}} a\right)\left|\alpha_{n}\right\rangle\left\langle\alpha_{n}\right|, \\
\mathbf{G}_{2}(a) & =-i \kappa \sum_{n} \frac{1}{\sqrt{\alpha_{n}}} \sinh \left(\sqrt{\alpha_{n}} a\right)\left|\alpha_{n}\right\rangle\left\langle\alpha_{n}\right|, \\
\mathbf{G}_{3}(a) & =\frac{i}{\kappa} \sum_{n} \sqrt{\alpha_{n}} \sinh \left(\sqrt{\alpha_{n}} a\right)\left|\alpha_{n}\right\rangle\left\langle\alpha_{n}\right| .
\end{aligned}
$$

The eigenmodes are the Bloch wave solutions and satisfy $\mathbf{H}_{1}\left|\alpha_{n}\right\rangle=\alpha_{n}\left|\alpha_{n}\right\rangle$. If the propagation wave vector $\vec{k}$ is at angle $\theta$ with respect to the $x y$-plane (see Figure 2, we are taking $\vec{k} \cdot \widehat{y}=0$ ), then the $\alpha_{n}$ are the solutions to

$$
\begin{array}{r}
\cos \left(\beta_{n} a^{\prime}\right) \cos \left(\gamma_{n}\left(\Lambda^{\prime}-a^{\prime}\right)\right)-\frac{1}{2}\left(\frac{\beta_{n}}{\gamma_{n}}+\frac{\gamma_{n}}{\beta_{n}}\right) \sin \left(\beta_{n} a^{\prime}\right) \sin \left(\gamma_{n}\left(\Lambda^{\prime}-a^{\prime}\right)\right) \\
=\cos \left(k \Lambda^{\prime} \sin \theta\right)
\end{array}
$$


where

$$
\beta_{n}=\sqrt{\kappa_{H}^{2}+\alpha_{n}}, \quad \text { and } \quad \gamma_{n}=\sqrt{\kappa_{L}^{2}+\alpha_{n}} .
$$

One can directly verify with eqs.(31) and (32), using the orthonormality and completeness properties of the eigenmodes, that $\mathbf{G}_{\mathrm{TE}}^{*}(a)=\mathbf{G}_{\mathrm{TE}}(-a)$ and $\mathbf{G}_{\mathrm{TE}}(a) \mathbf{G}_{\mathrm{TE}}(-a)=\mathbf{1}$ as required by propagation reciprocity.

The expression for the characteristic matrix for the second layer is similar. The components of eq.(31) are

$$
\begin{aligned}
\mathbf{G}_{1}(\Lambda-a) & =\sum_{n} \cosh \left(\sqrt{\alpha_{n}^{\prime}}(\Lambda-a)\right)\left|\alpha_{n}^{\prime}\right\rangle\left\langle\alpha_{n}^{\prime}\right|, \\
\mathbf{G}_{2}(\Lambda-a) & =-i \kappa \sum_{n} \frac{1}{\sqrt{\alpha_{n}^{\prime}}} \sinh \left(\sqrt{\alpha_{n}^{\prime}}(\Lambda-a)\right)\left|\alpha_{n}^{\prime}\right\rangle\left\langle\alpha_{n}^{\prime}\right|, \\
\mathbf{G}_{3}(\Lambda-a) & =\frac{i}{\kappa} \sum_{n} \sqrt{\alpha_{n}^{\prime}} \sinh \left(\sqrt{\alpha_{n}^{\prime}}(\Lambda-a)\right)\left|\alpha_{n}^{\prime}\right\rangle\left\langle\alpha_{n}^{\prime}\right| .
\end{aligned}
$$

The $\left|\alpha_{n}^{\prime}\right\rangle$ are eigenstates of $\mathbf{H}_{1}$ with $\epsilon(x)$ the profille in the $z=a$ to $\Lambda-a$ slab. Furthermore, the $\alpha_{n}^{\prime}$ satisfy the same equation, eq.(33), when we replace $\beta_{n}$ with $\beta_{n}^{\prime}$ and $\gamma_{n}$ with $\gamma_{n}^{\prime}$, where

$$
\beta_{n}^{\prime}=\sqrt{\kappa_{L}^{2}+\alpha_{n}^{\prime}}, \quad \text { and } \quad \gamma_{n}^{\prime}=\sqrt{\kappa_{H}^{2}+\alpha_{n}^{\prime}} .
$$

The characteristic matrix for the two layers together is

$$
\mathbf{G}_{\mathrm{TE}}(\Lambda)=\mathbf{G}_{\mathrm{TE}}(\Lambda-a) \mathbf{G}_{\mathrm{TE}}(a)
$$

Plugging in eqs.(32) and (35), the diagonal elements of eq.(37) are

$$
\begin{aligned}
\left(\mathbf{G}_{\mathrm{TE}}(\Lambda)\right)_{11} & =\sum_{m} \sum_{n}\left[\cosh \left(\sqrt{\alpha_{n}} a\right) \cosh \left(\sqrt{\alpha_{m}^{\prime}}(\Lambda-a)\right)\right. \\
& \left.+\sqrt{\frac{\alpha_{n}}{\alpha_{m}^{\prime}}} \sinh \left(\sqrt{\alpha_{n}} a\right) \sinh \left(\sqrt{\alpha_{m}^{\prime}}(\Lambda-a)\right)\right]\left|\alpha_{m}^{\prime}\right\rangle\left\langle\alpha_{m}^{\prime} \mid \alpha_{n}\right\rangle\left\langle\alpha_{n}\right| \\
\left(\mathbf{G}_{\mathrm{TE}}(\Lambda)\right)_{22} & =\sum_{m} \sum_{n}\left[\cosh \left(\sqrt{\alpha_{n}} a\right) \cosh \left(\sqrt{\alpha_{m}^{\prime}}(\Lambda-a)\right)\right. \\
& \left.+\sqrt{\frac{\alpha_{m}^{\prime}}{\alpha_{n}}} \sinh \left(\sqrt{\alpha_{n}} a\right) \sinh \left(\sqrt{\alpha_{m}^{\prime}}(\Lambda-a)\right)\right]\left|\alpha_{m}^{\prime}\right\rangle\left\langle\alpha_{m}^{\prime} \mid \alpha_{n}\right\rangle\left\langle\alpha_{n}\right|
\end{aligned}
$$

Following the procedure outlined in eqs.(2) to (4) (keep in mind that $\mathbf{1}=$ $\left.\sum_{n}\left|\alpha_{n}\right\rangle\left\langle\alpha_{n}\right|\right)$, the final spectral equation for the crystal in Figure 2 is

$$
\cosh \left(\sqrt{\alpha_{n}} a\right) \cosh \left(\sqrt{\alpha_{m}^{\prime}}(\Lambda-a)\right)
$$




$$
\begin{array}{r}
-\frac{1}{2}\left(\sqrt{\frac{\alpha_{n}}{\alpha_{m}^{\prime}}}+\sqrt{\frac{\alpha_{m}^{\prime}}{\alpha_{n}}}\right) \sinh \left(\sqrt{\alpha_{n}} a\right) \sinh \left(\sqrt{\alpha_{m}^{\prime}}(\Lambda-a)\right) \\
=\cos (k \Lambda \cos \theta) .
\end{array}
$$

Eq.(33), (33) with substitution eq.(36), and eq.(39) constitute the complete set of spectral band equations for TE polarization for the 2-D crystal in Figure 2. For a given $k, \theta$, and $\kappa=\omega / c$, eq.(33) determines the $\alpha_{n}$, and eq.(33) with the substitution eq.(36) deteremines the $\alpha_{m}^{\prime}$. However, only the combination of $\alpha_{n}$ and $\alpha_{m}^{\prime}$ that satisfy eq.(39) will propagate.

We acquired quite a bit of experience in efficiently finding solutions to equations of the type in eq.(33) when working with volume gratings[5]. Using this background, we developed and implemented an iterative algorithm to find the simultaneous solution $\omega$ to the three spectral equations given $k$ and $\theta$. The input fixes the right-hand side of eq.(39) and tells us which surface $\alpha_{n}$ and $\alpha_{m}^{\prime}$ must reside on. We start by choosing an $\alpha_{n}$, then determine the $\alpha_{m}^{\prime}$ that satisfies eq.(39). Changes to $\alpha_{n}$ and $\alpha_{m}^{\prime}$ that we make in iteration must stay on this surface. The proper change in $\alpha_{m}^{\prime}$ given a change in $\alpha_{n}$ is obtained by setting the total differential of eq.(39) to zero. With $\alpha_{n}$ and $\alpha_{m}^{\prime}$, we find the $\omega_{1}$ and $\omega_{2}$ respectively that satisfy eq.(33) and the modification with eq.(36). When $\omega_{1}=\omega_{2}$ we have found a solution. To get closer on the next iteration, we change $\omega_{1}$ by $\Delta \omega_{1}$ and $\omega_{2}$ by $\Delta \omega_{2}$ such that $\omega_{1}+$ $\Delta \omega_{1}=\omega_{2}+\Delta \omega_{2}$. The change in $\alpha_{n}$ resulting from $\Delta \omega_{1}$ is obtained from the derivative of eq.(33). The change in $\alpha_{m}^{\prime}$ from $\Delta \omega_{2}$ follows from the derivative of eq.(33) with the eq.(36) modification. These are plugged back into the total differential of eq.(39). Using $\omega_{1}+\Delta \omega_{1}=\omega_{2}+\Delta \omega_{2}$, this can be written as a linear equation in $\Delta \omega_{1}$ which we solve for. The new value for $\omega_{1}$ is used to find the new value in $\alpha_{n}$, and the process is started over again. The iterations converge rapidly, especially when the initial iteration is based on the solution to a nearby value of $k$ and $\theta$.

We compared these results with those obtained by the "established" numerical methods. However, the established methods involving truncated Fourier series, while our results are exact and analytical with no truncation in any form. Thus, the comparison actually amounts to using our results to verify that enough Fourier terms were included to obtain accurate results in the established methods. For TE polarization, the results indicate that 20 terms are usually sufficient. However, more terms are needed to get an accurate representation of the eigenvectors $\left|\alpha_{n}\right\rangle$ and $\left|\alpha_{m}^{\prime}\right\rangle$. In our approach, exact eigenvectors for a given $\alpha_{n}$ or $\alpha_{m}^{\prime}$ are found by solving eqs.(10) and 
(11).

For TM polarization, the magnetic field is linearly polarized in the $y$ direction: $\vec{H}(x, z)=H_{y}(x, z) \widehat{y}$. Maxwell's equations can be written as

$$
\partial_{z}\left(\begin{array}{c}
H_{y} \\
E_{x}
\end{array}\right)=\mathbf{H}_{\mathrm{TM}}\left(\begin{array}{c}
H_{y} \\
E_{x}
\end{array}\right),
$$

where

$$
\mathbf{H}_{\mathrm{TM}}=-\frac{i}{\kappa \epsilon}\left(\begin{array}{cc}
0 & -\kappa^{2} \epsilon^{2} \\
\mathbf{H}_{2} & 0
\end{array}\right),
$$

and

$$
\mathbf{H}_{2}=-\partial_{x}^{2}+\partial_{x}(\ln \epsilon) \partial_{x}-\kappa^{2} \epsilon(x) .
$$

For the photonic crystal illustrated in Figure 2, the derivative of the dielectric in the $x$-direction is zero except at the block boundaries at $x=0, a^{\prime}, \Lambda^{\prime}, \ldots$ where it is proportional to a $\delta$-function. The presence of the $\delta$-function in $\mathbf{H}_{2} H_{y}$ indicates that the derivative of $H_{y}, \partial_{x} H_{y}$ is discontinuous at the dielectric boundaries. The size of the discontinuity can be determined directly for $\mathbf{H}_{2} H_{y}$ by integrating it in the $x$-direction. The discontinuity in the derivative of $H_{y}$ is identical with the boundary conditions for Maxwell's equations that require the $z$-component of the electric field to be continuous. Since $E_{z}=(-i / \kappa) \partial_{x} H_{y} / \epsilon$, and $\epsilon$ is discontinuous, then $\partial_{x} H_{y}$ is discontinuous. The $\partial_{x} \ln \epsilon$ term in $\mathbf{H}_{2}$ reflects exactly this.

The characteristic matrix for TM polarization, $\mathbf{G}_{T M}(z)=\exp \left(\mathbf{H}_{T M} z\right)$, as in TE polarization, can be expressed in block matrix form[1]:

$$
\mathbf{G}_{\mathrm{TM}}=\left(\begin{array}{ll}
\mathbf{G}_{1} & \mathbf{G}_{2} \\
\mathbf{G}_{3} & \mathbf{G}_{4}
\end{array}\right),
$$

where each submatrix is expressed as a sum over the TM eigenmodes $\left|\alpha_{n}\right\rangle$ :

$$
\begin{aligned}
\mathbf{G}_{1}(z) & =\sum_{n} \cosh \left(\sqrt{\alpha_{n}} z\right)\left|\alpha_{n}\right\rangle\left\langle\alpha_{n}\right| \\
\mathbf{G}_{2}(z) & =i \kappa \sum_{n} \frac{1}{\sqrt{\alpha_{n}}} \sinh \left(\sqrt{\alpha_{n}} z\right)\left|\alpha_{n}\right\rangle\left\langle\alpha_{n}\right| \epsilon, \\
\mathbf{G}_{3}(z) & =-\frac{i}{\kappa} \sum_{n} \sqrt{\alpha_{n}} \sinh \left(\sqrt{\alpha_{n}} z\right) \frac{1}{\epsilon}\left|\alpha_{n}\right\rangle\left\langle\alpha_{n}\right|, \\
\mathbf{G}_{4}(z) & =\sum_{n} \cosh \left(\sqrt{\alpha_{n}} z\right) \frac{1}{\epsilon}\left|\alpha_{n}\right\rangle\left\langle\alpha_{n}\right| \epsilon .
\end{aligned}
$$


The eigenmodes are the Bloch wave solutions and satisfy $\mathbf{H}_{2}\left|\alpha_{n}\right\rangle=\alpha_{n}\left|\alpha_{n}\right\rangle$, and satisfy the spectral equation (for $z$ between 0 and $a$ ):

$$
\begin{array}{r}
\cos \left(\beta_{n} a^{\prime}\right) \cos \left(\gamma_{n}\left(\Lambda^{\prime}-a^{\prime}\right)\right)-\frac{1}{2}\left(\frac{\beta_{n} \epsilon_{L}}{\gamma_{n} \epsilon_{H}}+\frac{\gamma_{n} \epsilon_{H}}{\beta_{n} \epsilon_{L}}\right) \sin \left(\beta_{n} a^{\prime}\right) \sin \left(\gamma_{n}\left(\Lambda^{\prime}-a^{\prime}\right)\right) \\
=\cos \left(k \Lambda^{\prime} \sin \theta\right),(45)
\end{array}
$$

where $\beta_{n}$ and $\gamma_{n}$ are given by eq,(34). It is important to notice that the differential operator $\mathbf{H}_{2}$ in eq.(42) is not self-adjoint with respect to the usual inner product,

$$
\langle f \mid g\rangle=\frac{1}{\Lambda^{\prime}} \int_{0}^{\Lambda^{\prime}} d x f^{*}(x) g(x) .
$$

Thus, the eigenmodes with the inner product eq.(46) (which is used in the TE case) are not orthogonal. One could construct from the non orthogonal set of eigenmodes a basis that is orthogonal using the Gram-Schmidt procedure, but this would be unsatisfactory and unworkable as the new basis elements are no longer eigenstates and therefore not solutions to Maxwell's equations.

However, $\mathbf{H}_{2}$ can be put into Sturm-Liouville format. From this one can see that $\mathbf{H}_{2}$ is self-adjoint with respect to the inner product

$$
\langle f \mid g\rangle_{\epsilon}=\frac{1}{\Lambda^{\prime}} \int_{0}^{\Lambda^{\prime}} d x \frac{1}{\epsilon(x)} f^{*}(x) g(x) .
$$

With this inner product, the TM eigenmodes are orthonormal, $\left\langle\alpha_{m} \mid \alpha_{n}\right\rangle_{\epsilon}=$ $\delta_{m n}$. This is the inner product that must be used in calculations involving the characteristic matrix $\mathbf{G}_{T M}$, eq.(44).

Interestingly, $\left\langle\alpha_{m} \mid \alpha_{n}\right\rangle_{\epsilon}=\delta_{m n}$ is the correct procedure for normalizing eigenvectors. Most numerical routines normalize the eigenvectors in the process of computing them. "Canned" routines will use eq.(46), thus the resulting eigenvectors will not be normalized correctly. Furthermore, the correct Fourier expansion also requires use of the $\langle\mid\rangle_{\epsilon}$ inner product. Using the wrong inner product will mean that many more "Fourier" terms will be needed to get accurate eigenvalues, and that the Fourier coefficients will have no physical meaning. We have found that it takes more than 50 Fourier modes (computed with the inner product eq.(46)) to begin to see that the TM eigenvectors are indeed orthogonal under the correct $\langle\mid\rangle_{\epsilon}$ inner product.

At this point, using the proper inner product, the analysis is very similar to the TE case, and the correspondence between eq.(45) for the TM case, and eq.(33) for the TE case carries through for the remaining spectral equations. 


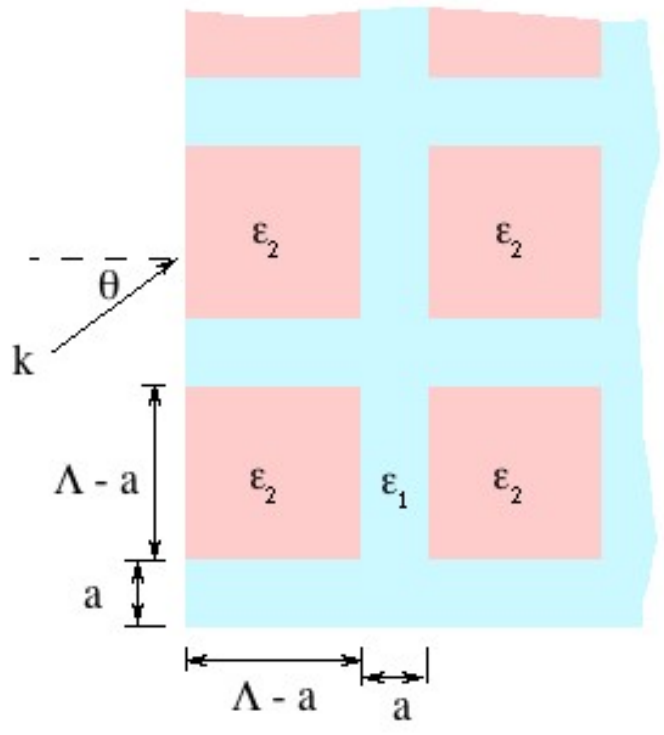

Figure 3: Unit cell of a 2-D photonic crystal made from a square lattice of dielectric veins.

As a quick but interesting example, and to further demonstrate the use of characteristic matrices, consider the crystal in Figure 3 featuring a square lattice with dielectric veins. We can obtain the spectral equations from the results we have already obtained. The first layer is identical to that in the crystal in Figure 2 with $a^{\prime} \rightarrow a$ and $\Lambda^{\prime} \rightarrow \Lambda$ and width equal to $\Lambda-a$. Thus the first spectral equation is given by eq.(33) and is

$$
\begin{aligned}
\cos \left(\beta_{n} a\right) \cos \left(\gamma_{n}(\Lambda-a)\right)-\frac{1}{2}\left(\frac{\beta_{n}}{\gamma_{n}}+\frac{\gamma_{n}}{\beta_{n}}\right) \sin \left(\beta_{n} a\right) & \sin \left(\gamma_{n}(\Lambda-a)\right) \\
& =\cos (k \Lambda \sin \theta)
\end{aligned}
$$

where $\beta_{n}$ and $\gamma_{n}$ are still given by eq.(34). The result for the second layer is the same as the first layer in Figure 2 in the limit $\Lambda^{\prime}-a^{\prime} \rightarrow 0$. This result is

$$
\cos \left(\beta_{n} \Lambda\right)=\cos (k \Lambda \sin \theta) \quad \text { or } \quad \beta_{n}=k \Lambda \sin \theta,
$$

which implies

$$
\alpha_{n}^{\prime}=k^{2} \sin ^{2} \theta-\kappa_{H}^{2},
$$


which is just transverse phase matching at the boundary (Snell's Law). The final equation is obtained from eq.(39) by interchanging $\alpha_{n}$ with $\alpha_{m}^{\prime}$ and using eq.(50) for $\alpha_{m}^{\prime}$. We compared the resulting dispersion relations and photonic bands and gaps with those in Reference 3, and found them in agreement. Of course, we repeat again that out results are exact and analytical, thus we are really verifying the numerical approach the authors of Reference 3 are using.

We implemented the spectral equation solver with a web-user interface for imput and output. Access is through a form on a browser. The user inputs the dielectric values, $\epsilon_{1}$ and $\epsilon_{2}$, the wavevector of the wave, $k$, and its angle of incidence, $\theta$ with respect to the $z$-axis, the size of the blocks, $a$, and the period $\Lambda$. The input from the form is passed to a CGI program, where the eigenvalues $\alpha_{n}$ abd the frequency $\omega$ are computed. The results are then sent back to the browser (as an html file).

\section{Nonlinear Waveguides and Path Integrals}

In order to model second harmonic generation in waveguides, three plane waves are coupled together using a second-order susceptibility. Assume that the waveguide is oriented along the $z$-axis. The fields are assumed to be plane waves in the $z$-direction multipled by a complex amplitude:

$$
E_{j}(\vec{x}, t)=U_{j}(\vec{x}) \exp \left(-i\left(\omega_{j} t-\kappa_{j} z\right)\right),
$$

for $j=1$ to 3 . Using Maxwell's equations, the three scalar fields are coupled together using a second-order susceptibility:

$$
\nabla^{2} E_{j}=\mu_{0} \epsilon \frac{\partial^{2}}{\partial t^{2}} E_{j}+\mu_{0} \chi_{j k l} \frac{\partial^{2}}{\partial t^{2}} E_{k} E_{l},
$$

where $j, k, l$ range from 1 to 3 , but all are distinct $(j \neq k \neq l)$, and where $\omega_{3}=\omega_{1}+\omega_{2}$. The plane-wave form, eq.(51) is inserted into the nonlinear Helmholtz equation (52). Taking the paraxial approximation, or equivalently, assuming only forward beam propagation, the complex amplitudes satisfy

$$
L_{j} U_{j}=\kappa_{j}^{2} \delta n^{2} U_{j}+\gamma \exp (-i \Delta(\kappa) z) U_{k} U_{l},
$$

where

$$
L_{j}=2 i \kappa_{j} \frac{\partial}{\partial z}+\nabla_{\perp}^{2}
$$

and $\Delta(\kappa)=\kappa_{3}-\kappa_{1}-\kappa_{2}$. The material constant $\gamma$ can have a frequency dependence, but this does not change the analysis any, so its explicit dependence is not important to the discussion. 
The conventional "established" (and unimaginative) approach to solving eq. (52) is to put the $U$ 's on a grid, express all derivatives as finite differences, and solve the resulting coupled algebraic equations. However, this ignores the fact that we know the inverse to the operator $L_{j}$ exactly, namely

$$
L_{j}^{-1}=G_{0}\left(\vec{x}-\vec{x}^{\prime}, z-z^{\prime}\right)=-\frac{i \kappa_{j}}{2 \pi\left(z-z^{\prime}\right)} \exp \left(i \frac{\kappa_{j}}{2} \frac{\left|\vec{x}-\vec{x}^{\prime}\right|^{2}}{z-z^{\prime}}\right) \theta\left(z-z^{\prime}\right),
$$

where $\theta(z)$ is the Heaviside step function. Furthermore, the free propagator $G_{0}$ is diagonal in momentum space.

When the equations (52) are put on a grid to formulate a finite difference system of equations, this is equivalent to placing all of the dielectric at the grid points, leaving vacuum in between. The propagation between grid points from $\left(x_{i}, y_{i}, z_{i}\right)$ to $\left(x_{j}, y_{j}, z_{i}+\Delta z\right)$ is done through the vacuum. Therefore, the propagation between points is accomplished using eq.(55). Using the propagator eq.(55) in effect carries far more orders than inverting the algebraic system of equations resulting from finite difference approximations to the derivatives. Furthermore, the nonlinear terms in eq.(53) just act as source terms.

The form of eq.(53) is very similar to the equations encountered in high energy laser propagation through the atmosphere. The form of eq.(53) is naturally set up to solve using a wave-optics simulation. To do this, the fields are defined on the $z=0$ plane. The fields are propagated to the next $x y$-plane at $\Delta z$ using eq.(55). As we mentioned, the propagator is diagonal in momentum space, hence the fields on a particular $x y$-plane are put through an FFT, the result multiplied point by point by the momentum space propagator, then the inverse FFT is taken. When the next $z$-plane is reached, the nonlinear terms are added, again by a simple point by point multiplication. One issue that arises in paraxial propagation is the possible aliasing of the phase front required by the paraxial propagator. However, this issue has long been solved in the laser propagation problem with an adaptive grid modification.

We modified our existing wave-optics simulation for laser propagation to simulate the three beam solution through a waveguide. The index crosssection profile consisted of a box of higher index material, width $a$, centered in the $x y$-plane. The grid was chosen to be large enough so that the there was no appreciable field amplitude at the edges. This is also standard procedure in atmospheric laser propagation simulations. The coeffciient $\gamma$ was allowed 
to vary periodically in the $z$ direction with a rectangular profile with period $\Lambda$. The simulation was set up to propagate over many periods in the $z$-direction. Initial conditions of flat plane waves, as well as fundamental modes were used. The fundamental modes were computed by requiring the beam profile to be constant in $z$ when $\gamma=0$. This is done in practice by propagating the beam back and forth in the z-direction until it does not change. For simple gradient profiles, it can be calculated analytically. Time constraints did not allow for a detailed comparison of results from our simulation with test cases, but the beam profiles and the power transfer to the signal beam agreed qualitatively with published results[e.g. 4] and simple analytical test cases.

Eq..(53) with $\gamma=0$ is mathematically identical to the $2+1 \mathrm{D}$ Schrödinger equation where $z$ plays the role of time. As discovered by R.P. Feynman, the propagator eq.(55) can be represented by a path integral,

$$
\int \mathcal{D} \vec{x}(z) \exp (i S[\vec{x}])
$$

where the integral represents the sum over all paths $\vec{x}(z)$ that start at $\left(x^{\prime}, y^{\prime}, z^{\prime}\right)$ and end at $(x, y, z)$. Each path is weighted by a phase factor $\exp (i S)$, where $S$ is the classical action for a free particle evaluated along the particular path,

$$
S[\vec{x}(z)]=\int_{z^{\prime}}^{z} d \bar{z} \frac{1}{2} \kappa\left(\partial_{\bar{z}} \vec{x}\right)^{2} .
$$

It can be shown that eq.(56) reproduces eq.(55) exactly[2], both when space is continuous, and when space has been discretized as we do in performing numerical calculations and simulations.

To find a path integral representation for the full nonlinear propagator, that is, for the Green's function solution of eq.(53), we sum over three different paths, $\vec{x}_{1}(z), \vec{x}_{2}(z)$, and $\vec{x}_{3}(z)$. The challenge, of course, is to find the proper (three particle) classical action. Fortunately, the nonlinear terms act as sources, and therefore

$S\left[\vec{x}_{1}, \vec{x}_{2}, \vec{x}_{3}\right]=\int_{z^{\prime}}^{z} d \bar{z} \frac{1}{2}\left[\kappa_{1}\left(\partial_{\bar{z}} \vec{x}_{1}\right)^{2}+\kappa_{2}\left(\partial_{\bar{z}} \vec{x}_{2}\right)^{2}+\kappa_{3}\left(\partial_{\bar{z}} \vec{x}_{3}\right)^{2}\right]+\gamma(\bar{z})\left|\vec{x}_{1}\right|\left|\vec{x}_{2}\right|\left|\vec{x}_{3}\right|$.

This is a completely new approach to analyzing waveguides. Time constraints prevented us from implementing an efficient numerical path summation algorithm, but the prospects are interesting. Usually, the path integral is dominated by several paths near the classical path (the least action path, 
commonly called "least time"). Given the purpose of a waveguide, those paths will lie near the center of the guide, forced there by the index profile. They can be obtained by aseveral methods Once a solution is obtained, no matter what method is used, the path integral is an excellent diagnostic tool

to analyze the power flow within the wave guide. This will be demonstrated in Phase 2.

\section{References}

1. S. Enguehard and B. Hatfield, "Characteristic matrices for diffraction gratings," AMP-01-03, preprint 2001.

2. B. Hatfield, Quantum Field Theory of Point Particles and Strings (Addison-Wesley, Redwood City, CA, 1992)

3. J. Joannopoulos, et al, Photonic Crystals, (Princeton University Press, 1995)

4. Chou, et al, Journal of Lightwave Technology, 16, 1686 (1998)

5. S. Enguehard and B. Hatfield, "An exact analytic solution to the coupled-wave analysis of planar diffraction gratings," AMP-01-04, preprint 2001. 